\title{
Erratum to: The effect of serotonin 1A receptor polymorphism on the cognitive function of premenstrual dysphoric disorder
}

\author{
Ju-Yu Yen $\cdot$ Hung-Pin Tu $\cdot$ Cheng-Sheng Chen $\cdot$ \\ Cheng-Fang Yen · Cheng-Yu Long • \\ Chih-Hung Ko
}

Published online: 23 November 2013

(c) Springer-Verlag Berlin Heidelberg 2013

\section{Erratum to: Eur Arch Psychiatry Clin Neurosci DOI 10.1007/s00406-013-0466-4}

In the original publication of the article contain some errors. The correct details are listed below.

The $5 \mathrm{THR}_{1 \mathrm{~A}}(\mathrm{rs} 6269)$ should be read as HTR1A(rs6295) in Table 1.

The HTR1A(rs6269) should be read as HTR1A(rs6295) in Table 1, Table 2 and Table 4.

The online version of the original article can be found under doi:10.1007/s00406-013-0466-4.

J.-Y. Yen · C.-S. Chen · C.-F. Yen · C.-H. Ko ( $\varangle)$ Department of Psychiatry, Faculty of Medicine, College of Medicine, Kaohsiung Medical University, Kaohsiung, Taiwan e-mail: cyberko@seed.net.tw; chihhungko@gmail.com

J.-Y. Yen · C.-S. Chen · C.-F. Yen · C.-H. Ko

Department of Psychiatry, Kaohsiung Municipal Ta-Tung

Hospital, Kaohsiung, Taiwan

J.-Y. Yen

Department of Psychiatry, Kaohsiung Medical University Hospital, Kaohsiung, Taiwan

H.-P. Tu

Department of Public Health and Environmental Medicine, Faculty of Medicine, College of Medicine, Kaohsiung Medical University, Kaohsiung, Taiwan

\section{C.-Y. Long}

Department of Obstetrics and Gynecology, Kaohsiung Municipal Hsiao-Kang Hospital, Kaohsiung Medical University,

Kaohsiung, Taiwan

\section{C.-H. Ko}

Department of Psychiatry, Kaohsiung Municipal Hsiao-Kang Hospital, Kaohsiung Medical University, Kaohsiung, Taiwan
The HTR1A rs6269 should be read as HTR1A rs6295 in part Figures (1D-F) labeling. The correct Fig. 1 has been given below. 

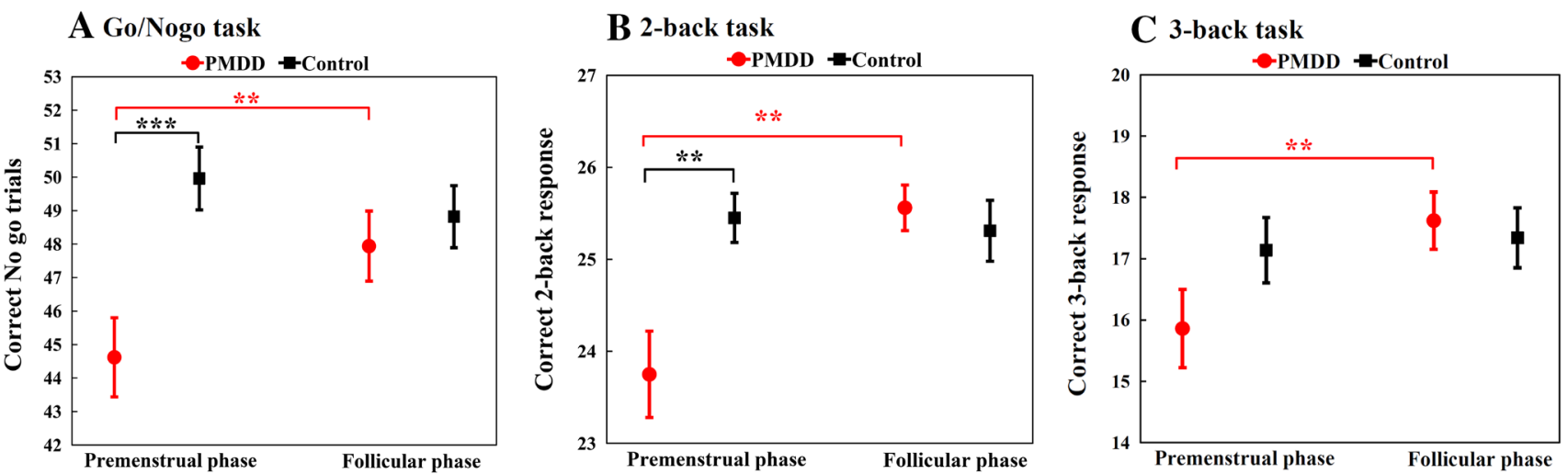

\section{Go/Nogo task}

E 2-back task

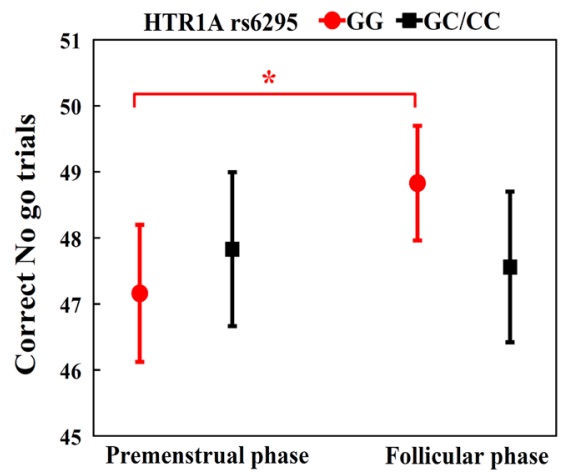

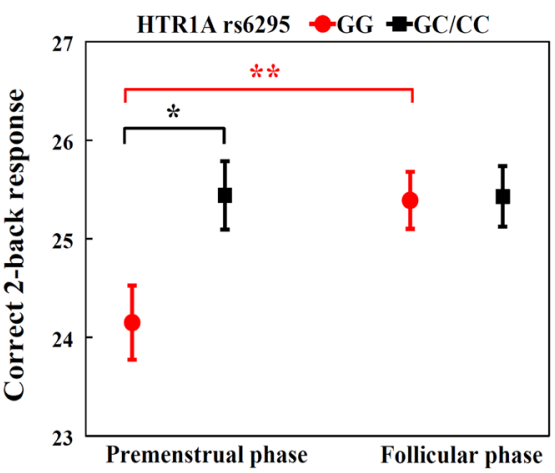

F 3-back task

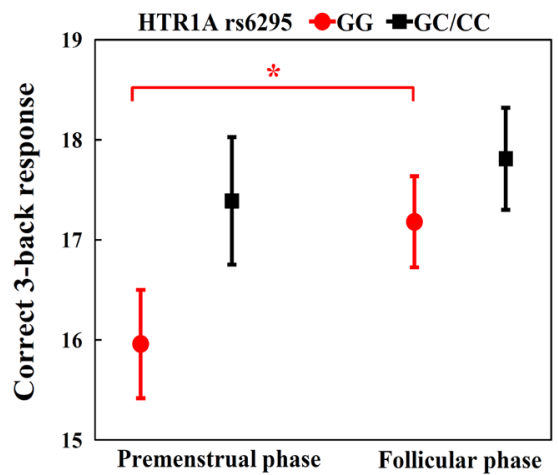

Fig. 1 The effects of premenstrual dysphoric disorder and the G/G genotype of serotonin receptor 1A (HTR1A; rs6295) on the performance in cognitive tasks across the menstrual cycle 